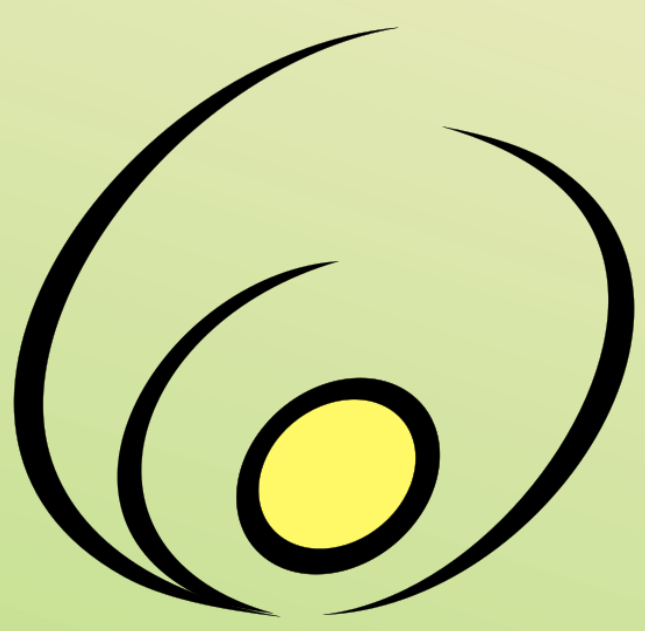

Fórum de

\section{Pró-Reitores}

\author{
de Extensão
}

\section{das Instituições}

\section{Públicas de}

\section{Educação Superior}

\section{Brasileiras}

Open access $\delta$ free available online

Revista Brasileira de Extensão Universitária

v. 9, n. 1, p. 17-25 jan.- abr. 2018 e-ISSN 2358-0399

DOI: https://doi.org/10.24317/2358-0399.2018v9i1.6799

\title{
Educação Continuada de Adultos: Noções de Alimentação Saudável e Manipulação de Alimentos
}

\author{
Izabella Alfaix Martins Palheiro Vicente ${ }^{1}$, \\ Giovanna Angela Leonel Oliveira ${ }^{2}$, Grazielle Gebrim Santos³, \\ Maria Luiza Rezende Ribeiro4, \\ Maria Aderuza Horst ${ }^{5}$, Mara Reis Silva ${ }^{6}$
}

Resumo: Considerando a demanda para o desenvolvimento de habilidades para geração de renda de famílias de comunidades carentes atendidas pelo Instituto Brasileiro de Benemerência e Integração do Ser (IBBIS), Goiânia, Estado de Goiás, Brasil, diagnosticou-se a necessidade de criar estratégias para compartilhar e fornecer conhecimentos básicos sobre produção, manipulação e consumo de alimentos, visando à promoção da saúde, aplicabilidade ocupacional e geração de renda. Assim, foram ministrados os cursos Noções Básicas de Higiene e Manipulação de Alimentos e Aproveitamento Integral dos Alimentos, para adultos de ambos os sexos de famílias de baixo poder aquisitivo, nas dependências do IBBIS. As aulas teóricas corresponderam a 30 a $40 \%$ e as práticas a 60 a $70 \%$ da carga horária total. Após a realização dos cursos, o desempenho dos participantes foi determinado por meio de testes oral e escrito. Por sua vez, os participantes responderam a questionários sobre os cursos e professores, contendo questões abertas e fechadas. O desempenho dos participantes foi satisfatório e houve ampla aprovação dos cursos, professores e conhecimentos compartilhados. Os cursos atenderam as expectativas dos participantes com relação à manipulação segura de alimentos e o aproveitamento de alimentos em preparações nutritivas.

Palavras-chave: Higiene, Alimentos, Educação, Segurança Alimentar e Nutricional. 


\title{
Continued education of adults: notions of healthy food and food manipulation
}

\begin{abstract}
Considering the demand for the development of skills for income generation of families from underprivileged assisted communities by the Instituto Brasileiro de Benemerência e Integração do Ser (IBBIS) in Goiânia city, Goiás State, Brazil, it was diagnosed the need to create strategies to share and provide basic knowledge about food production, manipulation and consumption, aiming at health promotion, occupational applicability and income generation. The courses of Basic Notions of Food Hygiene and Manipulation and Whole Food Utilization were given to adults of both sexes from low-income families in the dependencies of the IBBIS. The theoretical classes corresponded to 30 up $40 \%$ of the total course and practices, 60 to $70 \%$. After the end of the courses, the participant's performance was assessed through oral and written test. In turn, the participants answered the questionnaires, about the courses and teachers, containing direct and indirect questions. The performance of the participants was satisfactory and there was great approval of the courses, teachers and shared knowledge. The participants considered that the courses of safe food handling and the use of food in nutritional preparations were satisfactory.
\end{abstract}

Keywords: Food, Hygiene, Education, Food and Nutrition Security

Educación continua de adultos: conceptos de alimentación saludable y manipulación de alimentos

Resumen: Considerando la necesidad de desarrollar habilidades para generar renta de familias de comunidades carentes atendidas por el Instituto Brasileiro de Benemerência e Integração do Ser (IBBIS), Goiânia, Estado de Goiás, Brasil, se observó la necesidad de crear estrategias para compartir y proporcionar conocimientos básicos sobre producción, manipulación y consumo de alimentos, buscando promoción de la salud, aplicabilidad ocupacional y generación de renta. Se ofrecieron los cursos Nociones Básicas de Higiene y Manipulación de Alimentos y Aprovechamiento Integral de los Alimentos, para adultos de ambos géneros de familias de bajo poder adquisitivo en las dependencias del IBBIS. Las clases teóricas correspondieron a 30-40\% y las prácticas a 60-70\% de la carga horaria total. Después de la realización de los cursos, el desempeño de los participantes fue evaluado por medio de prueba oral y escrita. Los participantes respondieron los cuestionarios, sobre cursos y profesores, conteniendo cinco cuestiones abiertas y cerradas. El desempeño de los participantes fue satisfactorio y hubo amplia aprobación de los cursos, profesores y conocimientos compartidos por parte de los alumnos. Los cursos atendieron las expectativas de los participantes con relación a la manipulación segura de alimentos y el aprovechamiento de alimentos en preparaciones nutritivas.

Palabras-clave: Higiene, Alimentos, Educación, Seguridad Alimentaria y Nutricional

\section{Introdução}

O conhecimento sobre manipulação correta de alimentos e alimentação saudável auxilia a manutenção da saúde da família e é um dos itens essenciais para a elaboração e comercialização de alimentos prontos para o consumo. Os procedimentos incorretos de manipulação podem provocar Doenças Transmitidas por Alimentos (DTA), que são causadas por agentes infecciosos ou suas toxinas. Para assegurar a qualidade dos alimentos no aspecto higiênico e sanitário é primordial o conhecimento de diversos procedimentos, que têm início na compra da matéria-prima, e terminam com o consumo do alimento pronto. Tais cuidados visam à redução da probabilidade de contaminação química, física ou biológica (COSTA et al., 2013, BEZERRA; MANCUSO; HEITZ, 2014).

No Brasil houve aumento da prevalência de sobrepeso e obesidade, considerando os resultados de pesquisa do Estudo Nacional de Pesquisa Familiar (ENDEF) conduzido em 1974 pelo Instituto Brasileiro de Geografia e Estatística (IBGE), da Pesquisa de Vigilância de Fatores de Risco e Proteção para Doenças Crônicas por Inquérito Telefônico (VIGITEL) realizado em 2006 pelo Ministério da Saúde, e das Pesquisas de Orçamento Familiar (POF) realizadas pelo IBGE em parceria com o Ministério da Saúde nos anos 1995/1996, 2002/2003, 2008/2009 (HOBOLD; ARRUDA, 2015). O excesso de peso em homens $(18,5 \%)$ e mulheres $(28,7 \%)$ a partir de 20 anos, em 1974, aumentou gradativamente e em 2008/2009 alcançou 50,1\% e 48,0\%, respectivamente (IBGE, 2010).

O aumento da prevalência do excesso de peso dos brasileiros pode ser explicado pelas mudanças nos padrões de alimentação, especialmente entre as famílias com menor renda. Os alimentos preparados tradicionalmente com grãos e outros vegetais estão sendo substituídos por alimentos industrializados, que contêm teores elevados de gordura, açúcar e sal, e que são inferiores em qualidade nutricional quando comparados 
aos alimentos tradicionais. Estas mudanças do padrão alimentar favorecem a obesidade e o surgimento de outras doenças crônicas não transmissíveis (KEPPLE; SEGALL-CORREA, 2011).

A Organização Não Governamental (ONG) intitulada Instituto Brasileiro de Benemerência e Integração do Ser (IBBIS) é uma instituição filantrópica, sediada na Macrozona Rural do Barreiro de Goiânia, que tem como missão contribuir com a educação integral do ser humano, visando ao bem estar psíquico e social. Esta instituição atende aproximadamente 170 famílias de baixa renda por mês, residentes em bairros do Sudeste de Goiânia, e bairros vizinhos da cidade de Senador Canedo, oferecendo alimentação, atendimento médico e oficinas como de cabeleireiro, artesanato, manicure, pedicure e produção de horta. O IBBIS também dispõe de ônibus para transporte das pessoas das suas residências para a sede da instituição, com o propósito de facilitar a participação e o atendimento das famílias.

As famílias de baixo poder aquisitivo atendidas pelo IBBIS têm dificuldade de acesso ao serviço público de saúde, e necessitam de formação inicial e continuada para a capacitação em atividades que possam gerar renda. Muitas destas famílias vieram da região Norte do Brasil, em situação social, econômica e cultural deficitária, e com pouca informação sobre programas de aquisição de alimentos ou atendimento no sistema de saúde pública. A Faculdade de Nutrição da Universidade Federal de Goiás (FANUT/UFG), em parceria com o IBBIS, desenvolve projetos de formação inicial e continuada sobre segurança alimentar e nutricional. Tal atividade visa à promoção da saúde e o desenvolvimento de competências que auxiliem a geração de renda.

Considerando a demanda para o desenvolvimento de habilidades na área de alimentos e nutrição de famílias das comunidades carentes atendidas pelo IBBIS, diagnosticou-se a necessidade de criar estratégias para compartilhar e fornecer conhecimentos básicos sobre produção, manipulação e consumo de alimentos. Desse modo, o presente trabalho teve por objetivo promover ações de segurança alimentar e nutricional com ênfase no controle higienicossanitário dos alimentos, produção e aproveitamento de alimentos para famílias atendidas pelo IBBIS.

\section{Metodologia}

\section{Participantes}

Foram convidadas para participar do projeto vinte e cinco pessoas adultas de ambos os sexos, provenientes de famílias de baixa renda atendidas pelo IBBIS, residentes na região sudeste da cidade de Goiânia e bairros Morumbi, Jardim Aurora e Aurora das Mansões da cidade de Senador Canedo. O IBBIS considera famílias de baixa renda aquelas que habitam casas construídas com placas de alumínio, plástico ou alvenaria sem reboco, e sem condições adequadas de saneamento básico, e também as famílias cujos mantenedores recebam até um salário mínimo ou estejam desempregados. Apenas as pessoas com algum nível de alfabetização foram incluídas no projeto.

\section{Cursos realizados}

Os cursos sobre Noções Básicas de Higiene, Manipulação de Alimentos e Aproveitamento Integral dos Alimentos, com carga horária de seis horas cada, foram ministrados por docentes e acadêmicos do curso de Nutrição da UFG. Os cursos foram realizados nas dependências do IBBIS, em salas de aula e na cozinha, com a participação voluntária das pessoas. Desta forma, as aulas práticas corresponderam à aproximadamente $70 \%$ da carga horária total dos cursos.

O trabalho foi realizado em duas etapas, a primeira de outubro a novembro/2015, e a segunda de abril a maio/2016. Na primeira e na segunda etapa foram ministrados os dois cursos 'Noções Básicas de Higiene' e 'Manipulação de Alimentos e Aproveitamento Integral dos Alimentos', com carga horária total de 12 horas em cada etapa. A repetição dos cursos foi feita com a intenção de reforçar os conhecimentos básicos exigidos para uma pessoa que pretende trabalhar com a preparação de alimentos. Antes da segunda etapa, foram realizados aula de recuperação e teste escrito para os participantes que tiveram frequência igual ou inferior a $50 \%$.

\section{Conteúdo Programático e Recursos}

Foi utilizada metodologia participativa por meio de aulas teóricas e atividades práticas. As aulas foram ministradas com auxílio de recurso audiovisual (kit multimídia) para facilitar a discussão dos assuntos. No curso Noções Básicas de Higiene e Manipulação de Alimentos foram abordados temas sobre higiene pessoal, higiene de manipuladores de alimentos, controle higiênico e sanitário de locais, utensílios, equipamentos e alimentos (CECAN/RCO, 2002, ANVISA, 2004, SILVA JÚNIOR, 2005). Para reforçar o aprendizado realizou-se uma dinâmica, com os participantes, sobre higienização correta das mãos, utilizando água, sabão, papel toalha e solução de álcool $70 \%$.

No curso 'Aproveitamento Integral dos Alimentos' foram abordados temas sobre noções básicas de nutrição, alimentação saudável e aproveitamento integral de abóbora cabotiá, abacaxi, banana e melancia, em diversas receitas acessíveis ao poder aquisitivo dos participantes. Foram realizadas duas aulas práticas, com carga horária de duas horas cada, para a elaboração de receitas com os alimentos utilizados. Os participantes receberam material didático com a descrição, valor nutricional e custo das receitas.

\section{Avaliação dos participantes dos cursos}

Ao término do curso sobre higiene corporal, na primeira etapa, foi realizada uma avaliação oral das informações discutidas, com a premiação de 10 kits de higiene 
pessoal, para os participantes que acertassem uma pergunta sobre o assunto. A cada participante foi feita uma pergunta, caso a resposta estivesse incorreta, outra pergunta sobre o assunto era destinada ao mesmo participante, para uma segunda chance. Em caso de acerto, o participante recebia um kit de higiene pessoal.

Ao final da primeira etapa dos cursos foi organizada uma gincana, para avaliar o desenvolvimento de competências sobre higiene e manipulação de alimentos e aproveitamento dos alimentos e valor nutritivo.

$\mathrm{Na}$ primeira e na segunda etapa do trabalho foram realizadas avaliações escritas individuais dos participantes, através da atribuição de notas de zero a 10,0. Na avaliação objetiva, com cinco questões, foram explorados temas referentes à higiene corporal do manipulador de alimentos, boas práticas de manipulação, alimentação saudável e aproveitamento integral dos alimentos.

\section{Avaliação dos cursos e professores}

No final de cada curso, nas duas etapas, os participantes procederam à avaliação dos cursos e dos professores, com o uso de questionário próprio, com cinco questões, das quais três continham as alternativas ruim, boa, muito boa e excelente, e duas apresentavam as alternativas sim e não, além de uma questão dissertativa sobre comentários de aulas teóricas e práticas. Esta avaliação não era obrigatória.

\section{Análise dos resultados}

Foi feita uma análise descritiva das avaliações, por meio da frequência dos resultados do teste escrito e da avaliação do curso e dos professores feita pelos participantes da primeira e da segunda etapa do trabalho. Para os participantes que não atingissem 50\% da nota na avaliação escrita, e não tivessem $70 \%$ de frequência, estavam previstas atividades de recuperação, com novas atividades pedagógicas para promover a aprendizagem e reforçar as habilidades.

\section{Resultados e Discussão}

Primeira etapa - Cursos: Noções Básicas de Higiene e Manipulação de Alimentos e Aproveitamento Integral dos Alimentos

Durante a realização dos cursos, os participantes compartilharam as suas experiências em relação à higienização e acondicionamento de alimentos, além do uso de alimentos na preparação de receitas de valor nutritivo elevado. Embora, a maioria dos participantes demonstrasse conhecimento em relação às noções básicas de higiene pessoal, apenas uma pessoa tinha conhecimento sobre como higienizar corretamente as mãos, conforme a recomendação da Organização Mundial da Saúde (WHO, 2009). Algumas pessoas não sabiam como proceder para cuidar da higiene dos pés e axilas, para prevenir a proliferação de microrganismos, que causam odor desagradável e doenças, tais como micose e frieira. Além disso, não dominavam conhecimentos sobre Boas Práticas de Manipulação de Alimentos, em especial o descongelamento adequado dos alimentos e sanitização de hortaliças.

O desconhecimento ou negligência de Boas Práticas de Manipulação de Alimentos têm sido relatados em pesquisas desenvolvidas em cidades brasileiras. Em geral, os brasileiros não têm conhecimento adequado sobre segurança alimentar e isto pode ser associado com deficiência da educação formal, baixa renda familiar e falta de consciência sobre a responsabilidade deles na segurança alimentar (MOTTA, 2014, MEDEIROS; CARVALHO; FRANCO, 2017).

Lopes et al. (2015) avaliaram aspectos higienicossanitários de Unidades de Alimentação e Nutrição de 29 escolas municipais, da cidade de Bayeux no estado da Paraíba. Em relação aos manipuladores de alimentos, estes autores verificaram para a maioria deles vestuário inadequado, ausência de touca para proteção do cabelo, uso de adornos (pulseiras, anéis e brincos) e ausência da lavagem e assepsia das mãos antes da mudança de atividades com alimentos crus e preparados. Souza et al. (2015) pesquisaram as condições higiênicosanitárias de 30 pontos de comércio de alimentos em Uberaba, MG, e constaram que cerca de $40 \%$ dos manipuladores não tiveram cuidados pessoais com relação à higienização das mãos e uso de adornos. Foi também verificada a falta de cuidado com a higienização correta dos recipientes que acondicionavam alimentos. Motta (2014), em pesquisa com 2775 residências de 19 capitais brasileiras, concluiu que práticas inseguras de transporte, preparação e manipulação de sobras de alimentos são as mais associadas com o risco de contaminação.

A percepção do risco influencia o comportamento das pessoas sobre a manipulação higiênica dos alimentos, para controle da contaminação por agentes patogênicos. Consequentemente, a adoção de práticas adequadas para manipulação dos alimentos reduz os riscos para a saúde do consumidor (BRASIL et al., 2013, SOUZA et al., 2015). No entanto, a adesão das Boas Práticas de Manipulação de Alimentos deve ser efetivada por programas de capacitação permanente e campanhas educativas em segurança alimentar (MOTTA, 2014, SOUZA et al., 2015).

Considerando a importância do conhecimento sobre riscos na manipulação de alimentos e capacitação continuada sobre segurança alimentar, as informações básicas sobre higiene e manipulação foram utilizadas no curso subsequente - Aproveitamento Integral dos Alimentos - durante a elaboração dos alimentos. Os professores discutiram alimentação saudável e o valor nutritivo de bolos, biscoitos, farofa, suco e saladas elaboradas com abóbora cabotiá, banana, abacaxi e melancia.

Frutas e hortaliças contêm vitaminas, minerais, fibras e polifenóis, que são essenciais à manutenção da saúde humana (BLACK; BILLETTE, 2013). Os polifenóis, 
considerados compostos bioativos de alimentos, são encontrados naturalmente em frutas e hortaliças, e desempenham atividade antioxidante e outras ações biológicas importantes para redução do risco de doenças crônicas não transmissíveis (LUI, 2013). Dessa forma, o consumo desses alimentos promove a saúde. Ao contrário, uma alimentação inadequada é um dos fatores de risco para o desenvolvimento de doenças crônicas como a obesidade (VERDE, 2014). No entanto, as cascas, talos e sementes de frutas e hortaliças são normalmente desprezadas, embora muitas vezes possuam nutrientes e polifenóis em maior concentração, em comparação às partes destes alimentos consumida convencionalmente. Assim, o aproveitamento integral dos alimentos pode melhorar a qualidade nutricional da alimentação, auxiliar a promoção da saúde e reduzir o desperdício dos alimentos (STORCK et al., 2013, MALA; KURIAN, 2016).

No Brasil, tem sido observado modificações do padrão alimentar nas últimas décadas, com a constatação de consumo elevado de açúcar, sal, gordura e alimentos industrializados, e a redução da ingestão de arroz e feijão, além do consumo de frutas e hortaliças abaixo da recomendação (LINDEMANN; OLIVEIRA; MENDONZA-SASSI, 2016, GOMES; SILVA; CASTRO, 2017). Os participantes do curso declararam surpresa ao serem informados a respeito do conteúdo de açúcar e sal de alguns alimentos processados, consumidos com frequência, como refrigerantes e caldos de carne. Além disso, demonstraram interesse na elaboração de receitas com casca e sementes de alimentos.

Embora, a adesão às recomendações nutricionais e a mudança de hábito alimentar sejam difíceis de serem implementadas, as orientações feitas por profissionais de saúde podem favorecer o desenvolvimento de atitudes alimentares que promovam a saúde (LINDEMANN; OLIVEIRA; MENDONZA-SASSI, 2016).

\section{Primeira etapa - avaliação dos participantes, dos cursos e dos professores}

Para auxiliar a fixação de recomendações as avaliações dos cursos foram feitas com uma associação de teste oral, escrito e avaliação prática de caráter lúdico (gincana). $\mathrm{Na}$ primeira etapa, cerca de 25 pessoas participaram do curso sobre higiene pessoal e manipulação de alimentos. Entretanto, 10 participantes tiveram uma frequência baixa, pois se ausentaram de metade ou mais do total das aulas ministradas e cinco participantes desistiram dos cursos.

$\mathrm{Na}$ avaliação oral sobre higiene corporal todas as perguntas foram respondidas corretamente por 10 participantes voluntários, que receberam os kits de higiene contendo escova de dente, creme dental, sabonete, pente fino, barbeador e mini toalha de rosto.

A avaliação escrita, com questões dos temas higiene pessoal e manipulação de alimentos, foi respondida por 16 participantes, sendo que cerca de $56 \%$ obtiveram nota 9,5 e $37 \%$ nota 10 . Não houve nota abaixo de 8,0 , o que configura um aproveitamento elevado. A gincana realizada no final da primeira etapa teve a participação de cerca de 25 pessoas, distribuídas em dois grupos, que se revezaram nas respostas e dinâmicas sobre assuntos abordados nos dois cursos. A participação dos dois grupos foi motivada pelos professores e acadêmicos, e houve uma pequena diferença de pontuação entre os dois grupos.

Para os dez participantes que tiveram frequência igual ou inferior a $50 \%$ no curso Noções Básicas de Higiene e Manipulação de Alimentos, o curso foi repetido, e realizado um teste escrito. Sete pessoas fizeram a avaliação escrita, sendo que quatro obtiveram nota 10,0 , uma pessoa a nota 6,0 e duas receberam notas abaixo de 6,0. Apesar do empenho dos professores nas aulas de recuperação, alguns alunos não demonstraram interesse no aprendizado.

O questionário de avaliação do curso Noções Básicas de Higiene e Manipulação de Alimentos, e dos professores, referente à primeira etapa, foi respondido por apenas cinco participantes (Tabela 1), embora cerca de 25 pessoas tenham participado desse curso. A avaliação foi muito positiva, com declarações que o curso tinha oportunizado discussão de informações importantes sobre como cuidar do corpo humano. Por outro lado, vinte participantes do curso 'Aproveitamento Integral dos Alimentos' responderam o questionário e a maior parte deles avaliou as questões solicitadas como bom e ótimo (Tabela 1). Na quinta questão os participantes relataram que gostaram da oficina, gostaram dos professores e sugeriram que a oficina fosse realizada mais vezes e de maneira contínua.

Segunda etapa - Curso: Noções Básicas de Higiene e Manipulação de Alimentos e Aproveitamento Integral dos Alimentos

Após a primeira etapa, professores e administradores do IBBIS reavaliaram a estratégia de motivação dos participantes. A partir da segunda etapa, foi determinado para a aprovação dos participantes no curso, pelo menos $70 \%$ de frequência e nota mínima na avaliação escrita de 5,0. Para o recebimento de cesta básica, a aprovação no curso foi determinada como obrigatória pelos gestores do IBBIS. A mudança de estratégia influenciou positivamente a participação nos cursos de Noções Básicas de Higiene e Manipulação de Alimentos e Aproveitamento Integral dos Alimentos, com a inscrição de 20 pessoas, que frequentaram com maior assiduidade os cursos.

A entrega de cesta básica aos participantes que concluíram os cursos, por sugestão dos gestores do IBBIS, foi anunciada apenas no decorrer da segunda etapa do trabalho. No ato do convite esta condição não foi informada. Independentemente disto, as pessoas demonstraram maior interesse na participação das aulas teóricas e práticas e houve aumento da frequência às aulas. Os questionários de avaliação do curso e dos professores também foram respondidos por maior número de participantes. 
Tabela 1. Avaliação dos professores e dos cursos sobre 'Higiene e Manipulação de Alimentos' e 'Aproveitamento Integral dos Alimentos', da primeira etapa do trabalho, ministrados para adultos atendidos pelo Instituto Brasileiro de Benemerência e Integração do Ser, na cidade de Goiânia, GO (outubro a novembro/2015); os valores indicam número de respondentes.

\section{Curso: Noções Básicas sobre Higiene e Manipulação de Alimentos}

\begin{tabular}{|c|c|c|c|c|}
\hline Questões & Ruim & Bom & Muito bom & Excelente \\
\hline 1. Como você avalia o curso? & & & & 5 \\
\hline \multirow[t]{2}{*}{ 2. Como você avalia os professores? } & & & 1 & 4 \\
\hline & Sim & Não & Mais ou menos & \\
\hline 3. Este curso foi útil a você? & 5 & & & \\
\hline 4.Você recomendaria este curso a outra pessoa? & 5 & & & \\
\hline
\end{tabular}

Curso: Aproveitamento Integral dos Alimentos

\begin{tabular}{lcccc} 
& Ruim & Bom & Muito bom & Excelente \\
\cline { 2 - 4 } 1. Como você avalia o curso? & & 3 & 5 & 12 \\
2. Como você avalia os professores? & 3 & 4 & \\
\cline { 2 - 4 } & Sim & Não & Mais ou menos \\
\hline
\end{tabular}

3. Este curso foi útil a você?

20

4.Você recomendaria este curso a outra pessoa

20

Segunda etapa - avaliação dos participantes, dos cursos e dos professores

A avaliação escrita com questões dos temas higiene pessoal e manipulação de alimentos, alimentação saudável e aproveitamento integral dos alimentos foi feita por 19 participantes, sendo que apenas $10 \%$ dos participantes obtiveram nota 6,0 , e $90 \%$ alcançaram notas iguais ou superiores a 9,5. Isto evidencia o aproveitamento elevado dos conteúdos ministrados.

O curso Noções Básicas de Higiene e Manipulação de Alimentos e os professores foram avaliados por 12 participantes, sendo que a maioria deles atribuiu escores elevados às aulas ministradas e aos docentes, e todos consideraram o curso útil e o indicariam a outras pessoas (Tabela 2).

As pessoas fizeram comentários sobre a importância do curso e a qualidade dos professores e, em uma das avaliações foi solicitada alteração da distribuição das aulas. Alguns comentários, reproduzidos fielmente de acordo com a grafia dos participantes, foram: "As aulas são boas e esclarecedoras com professores interessados e dedicados a ensinar e esclarecer as dúvidas que surgem mas eu gostaria de ter mais aulas práticas do que somente teóricas, pois os assuntos às vezes são repetitivos e monótonos. Mas em geral o curso é muito útil e estou gostando"; "Me tirou algumas duvidas, do meu contidiano, principalmente em casa, como lida com os alimentos e utencilios domésticos, como lavar e cuidar"; "Agente aprendi muitas coisa importantes coisa que nois na maiorias não sabemos, e praticamos o que cada vez nos aprendi".

Os exemplos de comentários dos participantes demonstram que houve aprendizado sobre os assuntos abordados, embora para um dos participantes estes tenham sido repetitivos. Entretanto, existe uma recomendação geral, que a capacitação sobre higiene e manipulação de alimentos seja constante, pois é a percepção adequada do risco de contaminação que modifica o comportamento. Isto ocorre quando as 
pessoas incorporam novas atitudes, que contribuem para a segurança dos alimentos (BRASIL et al., 2013).

A avaliação do curso Aproveitamento Integral dos Alimentos e dos professores foi feita por 19 pessoas, sendo que cerca de $68 \%$ dos participantes atribuíram o escore excelente para as aulas e para os professores e todos consideraram úteis as informações deste curso e o indicariam a outra pessoa (Tabela 2). Alguns comentários foram feitos em relação ao aprendizado de novas informações: "Agora eu aproveito tudo, que tem de bom, nas frutas e verduras"; "Apredi a utiliza os alimentos que vão pro lixo"; "Aprendi como aproveitar igienizar os alimentos e como podemos melhorar os abitos alimentares".

A avaliação do desempenho dos participantes, dos cursos e dos professores realizada de maneira contínua e com feedback foi fundamental para a reformulação de atividades, que de outra forma poderiam prejudicar a troca de saberes ou a instrução da comunidade. Estes cursos devem ter continuidade para oportunizar a troca de saberes sobre manipulação, elaboração e consumo de alimentos saudáveis e nutritivos, e desse modo, permitir a interação entre comunidade e Universidade.

A expectativa com a realização dos cursos é de que os participantes incorporem o uso de alimentos de forma segura, quanto aos aspectos higienicossanitários. Estas práticas podem auxiliar a busca e obtenção de empregos em estabelecimentos que trabalham com a elaboração de alimentos. Além disso, os participantes dos cursos podem incorporar na própria alimentação preparações mais nutritivas, de baixo custo e que possam contribuir para as suas necessidades nutricionais.

Tabela 2. Avaliação dos professores e dos cursos sobre Higiene e Manipulação de Alimentos e Aproveitamento Integral dos Alimentos, da segunda etapa do trabalho, ministrados para adultos atendidos pelo Instituto Brasileiro de Benemerência e Integração do Ser, na cidade de Goiânia, GO (abril a maio/2016).

Curso: Noções Básicas sobre Higiene e Manipulação de Alimentos

\begin{tabular}{lcccc}
\hline Questões & Ruim & Bom & Muito bom & Excelente \\
\hline 1. Como você avalia o curso? & & 1 & 4 & 7 \\
2. Como você avalia os professores? & & 1 & 2 & \\
& & & & \\
& Sim & Não & Mais ou menos
\end{tabular}

3. Este curso foi útil a você?

12

4.Você recomendaria este curso a outra pessoa?

12

\section{Curso: Aproveitamento Integral dos Alimentos}

1. Como você avalia o curso?

2. Como você avalia os professores?

3. Este curso foi útil a você?

4.Você recomendaria este curso a outra pessoa?

\begin{tabular}{cccc} 
Ruim & Bom & Muito bom & Excelente \\
\hline 1 & 5 & 13 \\
& 1 & 5 & 13 \\
\hline Sim & Não & Mais ou menos \\
\hline
\end{tabular}

19

19 


\section{Conclusões}

As estratégias implementadas em uma comunidade de baixo poder aquisitivo, para compartilhar e fornecer conhecimentos sobre higiene corporal, manipulação e aproveitamento integral dos alimentos, foram adequadas ao nível de instrução dos participantes. Cursos com aulas teóricas e práticas, associadas ao uso de testes de avaliação oral e escrito, aulas de recuperação, gincana e formulários de avaliação de professores e dos cursos foram utilizados para promover troca de saberes e averiguar $\mathrm{o}$ aprendizado. As pessoas demonstraram interesse e participaram ativamente das aulas teóricas e práticas, especialmente quando foi exigida a frequência para o recebimento de benefício da instituição responsável. As avaliações do desempenho dos participantes foram satisfatórias, contudo os temas abordados necessitam de reciclagem constante. De maneira geral, os cursos atenderam as expectativas dos participantes com relação à manipulação segura de alimentos e o aproveitamento de alimentos em preparações nutritivas. Além disso, houve ampla aprovação dos cursos, dos professores e dos conhecimentos compartilhados. No entanto, os cursos devem ser aperfeiçoados com a ampliação da carga horária e a diversificação dos dias de realização, e do uso de outras estratégias para aumentar a participação das pessoas. Parcerias e convênios com estabelecimentos que servem refeições prontas, para oportunizar estágios e vagas de emprego aos participantes, são estratégias que podem auxiliar a geração de renda das famílias carentes.

\section{Contribuição de cada autor}

I.A.M.P.V., G.A.L.O. e G.G.S. auxiliaram o planejamento e a realização dos cursos, a elaboração de material didático, a análise de resultados e a redação do artigo; M.L.R.R e M.A.H. participaram da elaboração de material didático, da orientação dos alunos, da realização de palestras, das aulas práticas e da revisão do manuscrito, M.R.S. redigiu o projeto, participou do planejamento das atividades, da elaboração de material didático, da orientação de alunos, da realização de palestras e de aulas práticas, redigiu e revisou o manuscrito.

\section{Referências}

ANVISA. Cartilha sobre boas práticas para serviços de alimentação. 3. ed. Brasília: ANVISA, 2004. Disponível em: < http://portal.anvisa.gov.br/ documents/33916/389979/Cartilha+Boas+\%20Pr\%C3\% A1ticas+para+Servi\%C3\%A7os+de+Alimenta\%C3\%A7 \%C3\%A3o/d8671f20-2dfc-4071-b516-d59598701af0 > Acesso em: 10 ago. 2015.
BEZERRA, A. C. D.; MANCUSO, A. M. C.; HEITZ, S. J. J. Alimento de rua na agenda nacional de segurança alimentar e nutricional: um ensaio para a qualificação sanitária no Brasil. Ciência \& Saúde Coletiva, v. 19, n.5, p. 1489-1494, 2014.

BLACK, J. L.; BILLETTE, J. M. Do Canadians meet Canada's Food Guide's recommendations for fruits and vegetables. Applied Physiology, Nutrition and Metabolism, v. 38, n. 3, p. 234-42, 2013.

BRASIL, C. C. B.; HECKTHEUER, L. H. R.; GRESSLER, C. C.; MOURA, D. S.; PELEGRINI, S. B.; MEDEIROS, L. B. Conocimiento de los manipuladores de alimentos en el sector de los supermercados sobre higiene de los alimentos. Revista de Ciencia y Tecnología, v. 15, n. 20, 2013.

CECAN/RCO (CENTRO COLABORADOR EM ALIMENTAÇÃO E NUTRIÇÃO/ REGIÃO CENTROOESTE). Boas práticas de manipulação de alimentos para unidades de alimentação e nutrição. Goiânia: CECAN/RCO, 2002.

COSTA, J. N. P.; SANTOS, V. V. M.; SILVA, G. R.; MOURA, F. M. L.; GURGEL, C. A. B.; MOURA, A. P. B. L. Condições higiênico-sanitárias e físico-estruturais da área de manipulação de carne in natura em minimercados de Recife (PE), Brasil. Arquivos do Instituto Biológico, v. 80, n. 3, p. 352-358, 2013.

GOMES, F. S.; SILVA, G. A.; CASTRO, I. R. R. Aquisição domiciliar de refrigerantes e de biscoitos reduz o efeito de uma intervenção de promoção de frutas e hortaliças. Cadernos de Saúde Pública, v. 33, n. 3, e00023316, 2017.

HOBOLD, E.; ARRUDA, M. Prevalência de sobrepeso e obesidade em estudantes: relações com nível socioeconômico, sexo e idade. Revista Brasileira de Cineantropometria e Desempenho Humano, v.17, n.2, p.156-164, 2015 .

IBGE - INSTITUTO BRASILEIRO DE GEOGRAFIA E ESTATÍSTICAS. Pesquisa de Orçamentos Familiares no Brasil, 2008/2009 - antropometria e estado nutricional de crianças, adolescentes e adultos no Brasil. Rio de Janeiro: IBGE, 2010, 130 p.

KEPPLE, A.W; SEGALL-CORREA, A.M. Conceituando e medindo segurança alimentar e nutricional. Ciência \& Saúde Coletiva, v. 16, n. 1, p. 187-199. 2011.

LINDEMANN, I. L.; OLIVEIRA, R. R.; MENDONZASASSI, R. A. Dificuldades para alimentação saudável entre usuários da atenção básica em saúde e fatores associados. Ciência \& Saúde Coletiva, v. 21, n.2, p. 599-610, 2016.

LOPES, A. C. C.; PINTO, H. R. F.; COSTA, D. C. I. O.; MASCARENHAS, R. J.; AQUINO, J. S. Avaliação das Boas Práticas em unidades de alimentação e Nutrição de escolas públicas do município de Bayeux, PB, Brasil. Ciência \& Saúde Coletiva, v. 20, n.7, p. 2267-2275, 2015 . 
LUI, R.H. Health-promoting components of fruits and vegetables in the diet. Advances in Nutrition, v. 4, n. 3, p. 384S-392S, 2013.

MALA, K. S.; KURIAN, A. E. Nutritional composition and antioxidant activity of pumpkin wastes. International Journal of Pharmaceutical, Chemical and Biological Sciences, v. 6, n. 3, p. 336-344, 2016.

MEDEIROS, M. G. G. A.; CARVALHO, L. R.; FRANCO, R. M. Percepção sobre higiene dos manipuladores de alimentos e perfil microbiológico em restaurante universitário. Ciência \& Saúde Coletiva, v. 22, n.2, p. 383-392, 2017.

MOTTA, S. P. O. Consumer contribution to food contamination in Brazil: modelling the food safety risk en the home. Brazilian Journal and Food Technology, v. 17, n.2, p. 154-165, 2014.

SILVA JUNIOR, E. A. Manual de controle higiênicosanitário dos alimentos. 6. ed., São Paulo: Varela, 2005.

SOUZA, G. C.; SANTOS, C. T. B.; ANDRADE, A. A.; ALVES, L. Comida de rua: avaliação das condições higiênico-sanitárias de manipuladores de alimentos. Ciência \& Saúde Coletiva, v. 20, n.8, p. 2329-2338, 2015.

STORCK, C. R.; NUNES, G. L.; OLIVEIRA, B. B.; BASSO, C. Folhas, talos, cascas e sementes de vegetais: composição nutricional, aproveitamento na alimentação e análise sensorial de preparações. Ciência Rural, v. 43, n. 3, p. 537-543, 2013.

VERDE, S. M. M. L. Obesidade infantil: o problema de saúde pública do século XXI. Revista Brasileira em Promoção da Saúde, v. 27, n. 1, p.1-2, 2014.

WHO (WORLD HEALTH ORGANIZATION). Hand hygiene: why, how and when? Disponível em: < http://www.who.int/gpsc/5may/Hand_Hygiene_Why_Ho w_and_When_Brochure.pdf $>$. Acesso em: 10 ago. 2015.

\footnotetext{
Como citar este artigo:

VICENTE, I. A. M. P.; OLIVEIRA, G A. L.; SANTOS, G. G.; RIBEIRO, M. L. R.; HORST, M. A.; SILVA, M. R. Educação continuada de adultos: noções de alimentação saudável e manipulação de alimentos. Revista Brasileira de Extensão Universitária, v. 9, n. 1, p. 17-25, 2018. Disponível em: < https://periodicos.uffs.edu.br/index.php/RBEU/article/view/679 9/pdf $>$
} 